S.-M. Han · C. Namkoong • P. G. Jang • I. S. Park •

S. W. Hong - H. Katakami - S. Chun - S. W. Kim •

J.-Y. Park • K.-U. Lee • M.-S. Kim

\title{
Hypothalamic AMP-activated protein kinase mediates counter-regulatory responses to hypoglycaemia in rats
}

Received: 12 May 2005 / Accepted: 26 May 2005 / Published online: 23 August 2005

C) Springer-Verlag 2005

\begin{abstract}
Aims/hypothesis: Appropriate counter-regulatory hormonal responses are essential for recovery from hypoglycaemia. Although the hypothalamus is known to be involved in these responses, the molecular mechanisms have not been fully elucidated. AMP-activated protein kinase (AMPK) functions as a cellular energy sensor, being activated during energy depletion. As AMPK is expressed in the hypothalamus, an important site of neuroendocrine regulation, the present study was undertaken to determine whether hypothalamic AMPK mediates counter-regulatory responses to hypoglycaemia. Materials and methods: Hypoglycaemia was induced by i.p. injection of
\end{abstract}

Electronic supplementary material Supplementary material is available for this article at http://dx.doi.org/10.1007/s00125-0051913-1.

S.-M. Han · S. W. Kim · J.-Y. Park · K.-U. Lee · M.-S. Kim $(\bowtie)$

Department of Internal Medicine,

University of Ulsan College of Medicine,

Poongnap-dong, Songpa-gu,

Seoul, 138-736, South Korea

e-mail: mskim@amc.seoul.kr

Tel.: +82-2-30103245

Fax: $+82-2-30106962$

C. Namkoong · P. G. Jang · S. W. Kim

Asan Institute for Life Sciences,

University of Ulsan College of Medicine,

Seoul, South Korea

I. S. Park · S. W. Hong

Department of Anatomy, College of Medicine,

Inha University,

Incheon, Korea

H. Katakami

Department of Internal Medicine,

Miyazaki Medical College,

Miyazaki, Japan

S. Chun

Department of Laboratory Pathology,

University of Ulsan College of Medicine,

Seoul, South Korea regular insulin $(6 \mathrm{U} / \mathrm{kg})$ in Sprague-Dawley rats. Hypothalamic AMPK phosphorylation and activities were determined $1 \mathrm{~h}$ after i.p. insulin injection. To investigate the role of hypothalamic AMPK activation in mediating counter-regulatory responses, an AMPK inhibitor, compound $\mathrm{C}$, was pre-administered intracerebroventricularly (i.c.v.) or dominant-negative (DN)-AMPK was overexpressed in the hypothalamus before induction of hypoglycaemia. Results: Insulin-induced hypoglycaemia increased hypothalamic AMPK phosphorylation and $\alpha 2$ AMPK activities in rats. The change was significant in the arcuate nucleus/ventromedial hypothalamus (ARC/VMH) and paraventricular nuclei (PVN). Prior i.c.v. administration of compound $\mathrm{C}$ attenuated hypoglycaemia-induced increases in plasma concentrations of corticosterone, glucagon and catecholamines, resulting in severe and prolonged hypoglycaemia. ARC/VMH DN-AMPK overexpression impaired early counter-regulation, as evidenced by reduced glucagon and catecholamine responses. In contrast, PVN DN-AMPK overexpression attenuated late counter-regulation and corticosterone responses. Conclusions/ interpretation: Systemic hypoglycaemia causes hypothalamic AMPK activation, which is important for counterregulatory hormonal responses. Our data indicate that hypothalamic AMPK acts as a fuel gauge, sensing the whole-body energy state and regulating not only energy homeostasis but also neuroendocrine functions.

Keywords AMP-activated protein kinase Catecholamines $\cdot$ Corticosterone $\cdot$ Counter-regulatory response - Glucagon · Hypoglycaemia · Hypothalamus · Neuroendocrine - Paraventricular nucleus - Ventromedial hypothalamus

Abbreviations AICAR: 5-aminoimidazole-4carboxamide-riboside AMPK: AMP-activated protein kinase $\cdot$ ARC/VMH: arcuate nucleus/ventromedial hypothalamus - DN: dominant negative $\cdot$ HAAF: hypoglycaemia-associated autonomic failure $\cdot$ i.c.v.: intracerebroventricular $\cdot \mathrm{LH}$ : lateral hypothalamus $\cdot \mathrm{PVN}$ : paraventricular nucleus $\cdot$ SOX: supraoptic nucleus 


\section{Introduction}

Since the brain uses glucose as its primary energy source, hypoglycaemia is a serious concern for the brain. Prompt and coordinated counter-regulatory hormonal responses are thus critical for recovery from hypoglycaemia. Glucagon and sympathoadrenal activation is important in the early counter-regulatory responses to hypoglycaemia. On the other hand, cortisol and growth hormone responses, which occur after a prolonged and severe decrease in circulating glucose concentrations, are important for the long-term maintenance of blood glucose concentrations $[1,2]$.

It has long been thought that the hypothalamus, especially the ventromedial hypothalamus (VMH), is the key site of counter-regulatory responses. Local neuroglycopenia in the VMH has been shown to increase blood-glucose concentrations, together with marked elevations of plasma glucagon, epinephrine and norepinephrine [3]. In contrast, administration of glucose into the $\mathrm{VMH}$ prevented the elevation of glucagon and catecholamine during hypoglycaemia [4]. However, the molecular mechanisms mediating the counter-regulatory response remain unclear.

AMP-activated protein kinase (AMPK) is a heterotrimeric serine/threonine kinase that functions as a 'fuel gauge' in mammalian cells $[5,6]$. AMPK is activated during cellular energy depletion, switching off ATP-consuming pathways and switching on ATP-generating pathways. In humans and animals, AMPK activation has been associated with several key aspects of metabolism, including exercise-induced glucose uptake and fatty acid oxidation in skeletal muscle, cholesterol and fatty acid synthesis in the liver and glucose-mediated gene regulation in the pancreatic islets [7-9].

AMPK is also expressed in hypothalamic neurons involved in the regulation of energy homeostasis [10]. Recent studies have demonstrated that hypothalamic AMPK is activated by administration of the glucose anti-metabolite 2-deoxyglucose, and inhibited by administration of glucose $[11,12]$. Activation of hypothalamic AMPK causes an increase in food intake, which may act to restore depleted energy $[11,12]$.

Since the hypothalamus is the primary organ that regulates neuroendocrine function, hypothalamic AMPK may be involved in neuroendocrine regulation according to changes in the whole-body energy state. Therefore we investigated the changes in hypothalamic AMPK activity during insulin-induced hypoglycaemia and the role of hypothalamic AMPK in counter-regulatory responses.

\section{Materials and methods}

Animals

Male Sprague-Dawley rats weighing 300-350 g were maintained at ambient temperature $\left(22 \pm 1^{\circ} \mathrm{C}\right)$ and with 12:12 h light-darkness cycles (lights on 07.00 hours) with free access to water and rat chow unless otherwise indicated. Animals were purchased from Daehan Laboratory Animal Research (Seoul, Korea). All procedures followed the 'Principles of Laboratory Animal Care' (NIH, Washington, USA) and were approved by the Institutional Animal Care and Use Committee at the Asan Institute for Life Sciences (Seoul, Korea).

\section{Study design}

Study 1. Effect of insulin-induced hypoglycaemia on hypothalamic AMPK To induce hypoglycaemia, rats ( $n=11$ or 12 ) were given i.p. injections of $6 \mathrm{U} / \mathrm{kg}$ regular insulin (Humulin-R; Eli Lilly, Indianapolis, IN, USA) in the early light phase; as controls, rats were given saline vehicle. Rats were killed by decapitation $1 \mathrm{~h}$ later, and the medial hypothalamus or whole brain was collected and immediately frozen in liquid nitrogen.

Study 2. Effect of hypothalamic AMPK activation on counter-regulatory hormones To investigate the effect of hypothalamic AMPK activation on plasma concentrations of counter-regulatory hormones, a cannula was implanted into the third ventricle of each rat. Following a 7-day recovery period, rats that showed successful implantation of this intracerebroventricular (i.c.v.) cannula were randomly assigned into four groups. Rats were injected i.c.v. with vehicle (saline) or 1,10 or $100 \mathrm{nmol}$ of an AMPK activator, 5'-iminoimidazole-4-carboxamide ribonucleoside (AICAR), into the third ventricle in the early light phase. At $1 \mathrm{~h}$ after i.c.v. injection, rats were decapitated using a guillotine $(n=7)$. Trunk blood was collected in prechilled EDTA and heparin tubes containing $0.1 \mathrm{mg}$ aprotinin. Plasma was immediately separated by centrifugation for $5 \mathrm{~min}$ at $4^{\circ} \mathrm{C}$ and stored at $-70^{\circ} \mathrm{C}$ until assayed.

Animals were acclimatised to the procedure of injection and the guillotine apparatus, with sham decapitations performed daily for 3 days before the study, to minimise stress on the day of the study.

We also investigated the effect of hypothalamic AMPK activation on counter-regulatory hormones in hyperglycaemic or hypoglycaemic condition. Glucose $(2 \mathrm{~g} / \mathrm{kg})$ or insulin $(6 \mathrm{U} / \mathrm{kg})$ was i.p. administered $30 \mathrm{~min}$ before $100 \mathrm{nmol}$ i.c.v. AICAR $(n=8$ or 9$)$. One hour after i.c.v. injection, animals were killed and trunk blood was collected for measurement of plasma glucose, corticosterone, glucagon and catecholamine.

Study 3. Effect of a hypothalamic AMPK inhibitor (compound C) on insulin-induced hypoglycaemia To determine the effect of hypothalamic AMPK inhibition on insulin-induced hypoglycaemia, rats implanted with i.c.v. cannulae were randomly assigned into three groups $(n=9$ or 10). One group received sequential injection of i.c.v. DMSO with i.p. saline, the second i.c.v. DMSO with i.p. insulin, and the third i.c.v. injection of the AMPK inhibitor compound C (Merck, Rahway, NJ, USA) with i.p. insulin. 
In each animal, DMSO or compound C (100 nmol) was administered i.c.v. $1 \mathrm{~h}$ before i.p. injection of saline or insulin $(6 \mathrm{U} / \mathrm{kg})$ in the early light phase. Blood was taken from the tail vein at 30-min intervals until recovery from hypoglycaemia. Five days later, the rats received sequential i.c.v. and i.p. injections, as above, and were decapitated $1 \mathrm{~h}$ after i.p. injection. Trunk blood and the medial hypothalamus were collected. To exclude the possibility that compound $\mathrm{C}$ administered i.c.v. leaks into the systemic circulation and exerts peripheral action, another set of animals received i.p. compound C $(100 \mathrm{nmol}) 1 \mathrm{~h}$ prior to induction of hypoglycaemia $(n=5)$. Animals were decapitated $1 \mathrm{~h}$ after i.p. insulin injection and trunk blood was collected.

Study 4. Effect of dominant-negative AMPK overexpression in hypothalamic nuclei on insulin-induced hypoglycaemia To confirm the effect of hypothalamic AMPK inhibition on insulin-induced hypoglycaemia, rats were administered with an adenovirus expressing dominantnegative (DN)-AMPK. Adenovirus was injected into the bilateral ARC/VMH or the paraventricular nucleus (PVN), in which AMPK activation was caused by systemic hypoglycaemia in study 1 . Control rats were injected with adenovirus containing $\beta$-gal $(n=12-15)$. On the 4 th day after adenovirus injection, saline or insulin $(6 \mathrm{U} / \mathrm{kg})$ was administered i.p. during the early light period, and blood glucose was determined until the rats recovered from hypoglycaemia. On the fifth day after adenovirus injection, animals were killed $1 \mathrm{~h}$ after i.p. saline or insulin (6 U/kg) to collect the hypothalamus and trunk blood. Some of the animals were killed without injection to measure basal plasma counter-regulatory hormone.

Intracerebroventricular cannulation and injection

Stereotaxic implantation of an i.c.v. cannula aimed at the third cerebroventricle $(1.8 \mathrm{~mm}$ caudal to the bregma and $6.5 \mathrm{~mm}$ ventral to the sagittal sinus) was performed as described [13]. Following a 7-day recovery period, the animals were handled every day for 1 week to minimise stress. Correct positioning of the cannula was confirmed by a positive dipsogenic response to angiotensin II (150 ng). AICAR or compound $\mathrm{C}$ were dissolved in saline or DMSO and injected in a $10 \mu \mathrm{l}$ volume as described [13].

\section{Adenoviral gene transfer}

Plasmids encoding c-Myc-tagged forms of DN- $\alpha 1$-AMPK with a mutation altering Asp157 to alanine [14] and of DN- $\alpha 2$-AMPK with a mutation altering Lys45 to arginine [15] were the kind gifts of Dr J. Ha (Department of Molecular Biology, Kyunghee University College of Medicine, Seoul, Korea). Selective inhibition of $\alpha 2$-AMPK activity in the hypothalamus could increase $\alpha 1$-AMPK activity [12]. To effectively inhibit hypothalamic AMPK activity, we injected $900 \mathrm{nl}$ mixture $(1: 1 \mathrm{vol} / \mathrm{vol})$ of adenovirus expressing DN- $\alpha 1$-AMPK and DN- $\alpha 2$-AMPK $\left(10^{11}\right.$ plaque-forming units $\left.[\mathrm{pfu}] / \mathrm{ml}\right)$ with $100 \mathrm{nl}$ adenovirus expressing $\beta$-gal into the bilateral ARC/VMH $(9.2 \mathrm{~mm}$ depth, $2.8 \mathrm{~mm}$ post bregma, $0.6 \mathrm{~mm}$ lateral from the sagittal suture) and PVN (7.6 mm depth, $1.8 \mathrm{~mm}$ post bregma, $0.2 \mathrm{~mm}$ lateral from the sagittal suture) via a syringe pump (Harvard Apparatus, Holliston, MA, USA) at a rate of $100 \mathrm{nl} / \mathrm{min}$ for $10 \mathrm{~min}(1 \mu \mathrm{l} /$ injection site $)$ under ketamine $(100 \mathrm{mg} / \mathrm{kg})$ and xylazine $(20 \mathrm{mg} / \mathrm{kg})$ anaesthesia. Control rats were injected with $1 \mu \mathrm{l}$ adenovirus expressing $\beta$-gal $\left(10^{11} \mathrm{pfu} / \mathrm{ml}\right)$. The dose of adenovirus particles injected has been previously shown to effectively suppress AMPK activity [11]. The injection site was confirmed by $\beta$-gal staining, as described previously [16] (see Electronic supplementary material Fig. 1). Animals showing a proper location of $\beta$-gal staining were included in the data analysis.

Dissection of the hypothalamus

The medial hypothalamus was dissected in the anterior border of the optic chiasma, the posterior border of the mammillary body, the upper border of the anterior com-


Fig. 1 Effect of systemic hypoglycaemia on a phosphorylated (P-) and $\mathbf{b}$ total (T-) AMPK protein expression in the medial hypothalamus. Rats were killed by decapitation $1 \mathrm{~h}$ after i.p. injection of saline or insulin $(n=5$ or 6$) .{ }^{* *} p<0.005$ vs saline-injected control 
missure and the lateral border half way from the lateral sulcus in the ventral side of brain. Individual hypothalamic nuclei were dissected using a 'micropunch' technique as described [17]. Briefly, brain coronal sections $1 \mathrm{~mm}$ in thickness from the optic chiasma to the mammillary body were mounted onto glass slides. Using anatomical landmarks from a rat brain atlas [18], individual nuclei were punched out using 18-gauge stainless steel needles. The accuracy and reproducibility of the punches was established by inspecting the topography of the holes by transillumination under a low-power light microscope and by measuring the expression of selected transcripts.

\section{Measurement of AMPK activity}

The hypothalamus was incubated on ice for 2 min with digitonin buffer $(50 \mathrm{mmol} / \mathrm{l}$ Tris- $\mathrm{HCl}, \mathrm{pH} 7.3,50 \mathrm{mmol} / \mathrm{l}$ $\mathrm{NaF}, 30 \mathrm{mmol} / 1$ glycerol phosphate, $250 \mathrm{mmol} / 1$ sucrose, $1 \mathrm{mmol} / 1$ sodium metavanadate and $0.4 \mathrm{mg} / \mathrm{ml}$ digitonin) to lyse the cells. To measure isoform-specific AMPK activity, $40 \mu \mathrm{g}$ of each tissue lysate were immunoprecipitated by incubation with specific antibodies against the $\alpha 1-, \alpha 2$-and $\beta$-AMPK subunits (Upstate Biotechnology, Lake Placid, NY, USA) and $15 \mu \mathrm{l}$ of $25 \%$ (wt/vol) Protein G-Sepharose beads (Santa Cruz Biotechnology, Santa Cruz, CA, USA) overnight at $4^{\circ} \mathrm{C}$. AMPK activity was determined using the modified method of Davies et al. [19] with these fractionated proteins in kinase assay buffer (62.5 $\mathrm{mmol} / \mathrm{l}$
HEPES, pH 7.0, $62.5 \mathrm{mmol} / \mathrm{l} \mathrm{NaCl}, 62.5 \mathrm{mmol} / \mathrm{l} \mathrm{NaF}$, $6.25 \mathrm{mmol} / \mathrm{l}$ sodium pyrophosphate, $1.25 \mathrm{mmol} / 1 \mathrm{EDTA}$, $1.25 \mathrm{mmol} / 1 \mathrm{EGTA}$ and $1 \mathrm{mmol} / 1$ dithiothreitol) containing $200 \mu \mathrm{mol} / \mathrm{l}$ AMP, ATP mixture (200 $\mu \mathrm{mol} / 1$ ATP and $\left.1.5 \mu \mathrm{Ci}\left[\gamma_{-}{ }^{32} \mathrm{P}\right] \mathrm{ATP}\right)$, with or without $200 \mu \mathrm{mol} / 1 \mathrm{SAMS}$ peptide (HMRSAMSGLHLVKRR) at $30^{\circ} \mathrm{C}$ for $10 \mathrm{~min}$. The reaction was terminated by spotting the reaction mixture onto phosphocellulose paper (P81), which was extensively washed with $150 \mathrm{mmol} / 1$ phosphoric acid. The radioactivity was measured with a scintillation counter. The $\alpha 1$-and $\alpha 2$-AMPK activities were corrected by $\beta$-AMPK activity to analyse the effect of $\alpha 1$-and $\alpha 2-\mathrm{DN}-\mathrm{AMPK}$ overexpression.

\section{Western blot analysis}

Immunoblot analysis was conducted as previously described [20]. Forty-microgram samples of tissue lysate were separated by $8 \%$ SDS-PAGE and transferred to PVDF membranes. Following incubation in blocking buffer, the membranes were incubated overnight at $4^{\circ} \mathrm{C}$ with antibodies directed against the phosphorylated (Thr172) and total forms of AMPK (Cell Signaling, Beverly, MA, USA). Blots were developed using horseradish-peroxidase-linked anti-rabbit secondary antibody and a chemiluminescent detection system (New England Biolabs, Beverly, MA, USA). Band density was corrected in relation to the density of $\beta$-actin bands.
Fig. 2 Effect of systemic hypoglycaemia on a $\alpha 1$-AMPK and $\mathbf{b} \alpha 2$-AMPK activity in the medial hypothalamus. c $\alpha 2-$ AMPK activity in different hypothalamic areas. Rats were killed by decapitation $1 \mathrm{~h}$ after i.p. injection of saline or insulin $(n=5$ or 6$)$. $* p<0.05$ vs salineinjected control. Open bars, control group; filled bars, hypoglycaemic group

Table 1 Effect of i.c.v. administration of the AMPK activator AICAR on plasma counterregulatory hormone levels

Values are means \pm SEM $(n=7)$. ${ }^{\mathrm{a}} p<0.05$ vs saline-injected control rats

\begin{tabular}{lcccc}
\hline & Control & \multicolumn{2}{c}{ AICAR (mmol) } & \\
\cline { 3 - 5 } & & 1 & 10 & 100 \\
\hline Plasma glucose (mmol/l) & $5.7 \pm 0.3$ & $5.4 \pm 0.4$ & $6.2 \pm 0.5$ & $6.0 \pm 0.3$ \\
Plasma corticosterone (nmol/l) & $865.8 \pm 105.5$ & $1341.9 \pm 202.7$ & $1432.0 \pm 190.5$ & $2155.8 \pm 334.8^{\mathrm{a}}$ \\
Plasma glucagon (ng/l) & $31.5 \pm 3.3$ & $59.8 \pm 15.5$ & $67.1 \pm 28.0$ & $94.1 \pm 20.2^{\mathrm{a}}$ \\
Plasma epinephrine (nmol/l) & $13.3 \pm 4.0$ & $8.3 \pm 3.0$ & $20.5 \pm 7.1$ & $23.3 \pm 6.1$ \\
Plasma norepinephrine (nmol/l) & $60.4 \pm 17.2$ & $51.2 \pm 17.9$ & $74.2 \pm 19.9$ & $98.3 \pm 18.4$ \\
\hline
\end{tabular}

Measurement of blood samples

Blood-glucose concentrations were measured with an Acucheck glucometer (Roche, Milpitas, CA, USA). RIAs were used to measure plasma corticosterone (Diagnostic Product Corporation, Los Angeles, CA, USA) and glucagon (Linco Research, St Charles, MO, USA) concentrations. Plasma concentrations of epinephrine and norepinephrine were determined by HPLC using electrochemical detection (Hewlett Packard, Palo Alto, CA, USA).

Data analysis

All data are presented as means \pm SEM. Comparisons between groups were by Student's $t$-test or ANOVA followed by the post-hoc least significance difference test. Significance was defined as $p<0.05$.

\section{Results}

Insulin-induced hypoglycaemia activates hypothalamic $A M P K$ Intraperitoneal injection of insulin caused severe hypoglycaemia at $1 \mathrm{~h}$ after injection (saline $5.8 \pm 0.1$ vs insulin $1.6 \pm 0.1 \mathrm{mmol} / \mathrm{l})$. AMPK phosphorylation, an indicator of AMPK activation, was 1.7-fold higher in the hypothalamus of the hypoglycaemic group than in that of the control group (Fig. 1a), but total AMPK protein levels in the hypothalamus did not differ between the two groups (Fig. 1b). While $\alpha 1-A M P K$ activities were unaltered (Fig. 2a), $\alpha 2$-AMPK activities were increased 1.6-fold in the hypothalamus of the hypoglycaemic group (Fig. 2b). When we measured $\alpha 2$-AMPK activities in different hypothalamic areas, we found that hypoglycaemia increased these activities in the ARC/VMH and PVN by 1.7- and 2.4-fold, respectively (Fig. 2c). In contrast, there was no significant change caused in either the lateral hypothalamus (LH) area or the supraoptic nucleus (SOX).

Hypothalamic AMPK activation stimulates the counterregulatory responses Intracerebroventricular administration of the AMPK activator AICAR (10 and $100 \mathrm{nmol}$ ) in a euglycaemic condition significantly increased plasma corticosterone and glucagon concentrations at $1 \mathrm{~h}$ after injection. It also tended to increase plasma epinephrine and norepinephrine (Table 1). In the hyperglycaemic condition (plasma glucose $8.7 \mathrm{mmol} / 1$ ), i.c.v. adminis-
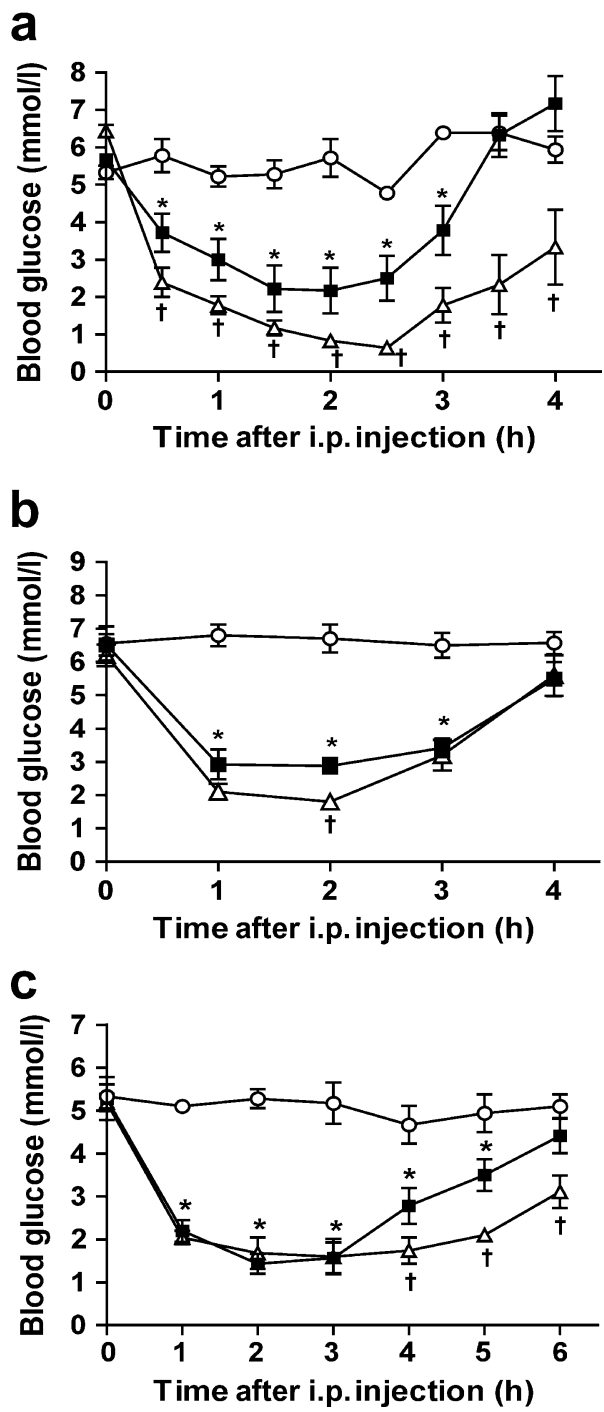

Fig. 3 Effect of hypothalamic AMPK inhibition on insulin-induced hypoglycaemia. a Effect of i.c.v. administration of compound $\mathrm{C}$ ( $n=9$ or 10$) .{ }^{*} p<0.05$ vs i.c.v. and i.p. vehicle-injected rats. ${ }^{\dagger} p<0.05$ vs i.c.v. vehicle-and i.p. insulin-injected rats. Open circles, i.c.v. DMSO with i. p. saline; solid squares, i.c.v. DMSO with i.p. insulin; open triangles, i.c.v. compound $\mathrm{C}$ with i.p. insulin. b, $\mathbf{c}$ Effect of DN-AMPK overexpression in ARC/VMH or PVN $(n=5-7)$. ${ }^{*} p<0.05$ vs intrahypothalamic $\beta$-gal-and i.p. saline-injected rats. ${ }^{\dagger} p<0.05$ vs intrahypothalamic $\beta$-gal-and i.p. insulin-injected rats. Open circles, i.c.v. ARC/VMH or PVN $\beta$-gal over expression with i.p. saline; solid squares, ARC/VMH or PVN DN-AMPK over expression with i.p. insulin; open triangles, $\mathrm{ARC} / \mathrm{VMH}$ or PVN with i.p. insulin 
Table 2 Effect of i.c.v. administration of the AMPK inhibitor compound $\mathrm{C}$ on counter-regulatory hormonal responses during hypoglycaemia

\begin{tabular}{lccc}
\hline & Control & Hypoglycaemia & $\begin{array}{l}\text { Hypoglycaemia } \\
\text { with i.c.v. } \\
\text { compound C }\end{array}$ \\
\hline $\begin{array}{l}\text { Plasma glucose } \\
\text { (mmol/l) }\end{array}$ & $5.7 \pm 9.2$ & $2.5 \pm 0.3^{*}$ & $1.2 \pm 0.2^{*}$ \\
$\begin{array}{l}\text { Plasma } \\
\text { corticosterone } \\
\text { (nmol/1) }\end{array}$ & $399.7 \pm 51.1$ & $886.8 \pm 130.0^{*}$ & $482.8 \pm 109.2^{\dagger}$ \\
$\begin{array}{l}\text { Plasma glucagon } \\
\text { (ng/l) }\end{array}$ & $78.7 \pm 15.3$ & $435.0 \pm 7.7^{*}$ & $263.9 \pm 38.3^{\dagger}$ \\
$\begin{array}{l}\text { Plasma } \\
\text { epinephrine } \\
\text { (nmol/l) }\end{array}$ & $39.1 \pm 4.2$ & $74.0 \pm 1.9^{*}$ & $41.8 \pm 8.7^{\dagger}$ \\
$\begin{array}{l}\text { Plasma } \\
\text { norepinephrine } \\
\text { (nmol/l) }\end{array}$ & $29.3 \pm 4.5$ & $53.8 \pm 12.6^{*}$ & $35.1 \pm 3.6^{\dagger}$ \\
$\begin{array}{l}\text { Hypothalamic } \\
\text { AMPK activity } \\
\text { (\% control) }\end{array}$ & $100 \pm 12$ & $166 \pm 26^{*}$ & $74.4 \pm 15^{\dagger}$ \\
\hline
\end{tabular}

Values are means \pm SEM ( $n=9$ or 10$)$

$* p<0.05$ vs control group

${ }^{\dagger} p<0.05$ vs hypoglycaemic group

tration of AICAR (100 nmol) increased plasma glucagon and epinephrine levels but paradoxically reduced plasma corticosterone levels (data not shown). In the hypoglycaemic condition (plasma glucose $2.2 \mathrm{mmol} / \mathrm{l}$ ), when hypothalamic AMPK activity is expected to be at a high level, i.c.v. AICAR did not further increase plasma counterregulatory hormone levels (data not shown).

Hypothalamic AMPK inhibition induces severe and prolonged hypoglycaemia In rats pre-administered i.c.v. compound C, i.p. insulin caused a more severe decrease in plasma glucose concentrations from 30 to $150 \mathrm{~min}$. Recovery from insulin-induced hypoglycaemia was significantly delayed in these rats (Fig. 3a). Two of these animals died without recovering from hypoglycaemia, whereas none of the animals in the control group died. In contrast, i.p. administration of compound $\mathrm{C}$ prior to i.p. insulin did not show any effect on hypoglycaemia (data not shown), suggesting that the effect of compound $\mathrm{C}$ was through the central nervous system.

To study the effect of region-specific AMPK inhibition, we overexpressed DN-AMPK in the PVN and ARC/ $\mathrm{VMH}$. In the ARC/VMH, DN-AMPK overexpression reduced the corrected $\alpha 1$-and $\alpha 2$-AMPK activities by 37 and $42 \%$ when compared with those of $\beta$-gal controls. In these rats, i.p. injection of the same amount of insulin caused more severe hypoglycaemia during the initial $2 \mathrm{~h}$ than in rats injected with $\beta$-gal adenovirus. However, the recovery time from hypoglycaemia was unaltered (Fig. 3b).

In the PVN, DN-AMPK overexpression reduced corrected $\alpha 1$-and $\alpha 2$-AMPK activities by 41 and $42 \%$, respectively. In contrast, in rats with bilateral PVN injection of DN-AMPK, there was no difference in the early phase of hypoglycaemia, whereas recovery from insulininduced hypoglycaemia was significantly delayed (Fig. 3c). Three of nine animals died during the late stage of hy-
Fig. 4 Effects of DN-AMPK overexpression in $\mathrm{ARC} / \mathrm{VMH}$ and PVN on plasma concentrations of a corticosterone, b glucagon, c epinephrine and d norepinephrine during hypoglycaemia. Rats were injected with adenovirus expressing $\beta$-gal or DN-AMPK into the bilateral $\mathrm{ARC} / \mathrm{VMH}$ or PVN $(n=5-7)$. On the fifth day of adenovirus injection, rats were killed $1 \mathrm{~h}$ after i.p. injection of saline or insulin. $* p<0.05$, ${ }^{* *} p<0.01$ between the indicated two groups. Open bars, $\beta$-gal with i.p. saline; filled bars, $\beta$-gal with i.p. insulin; hatched bars, DN-AMPK with i. p. insulin a

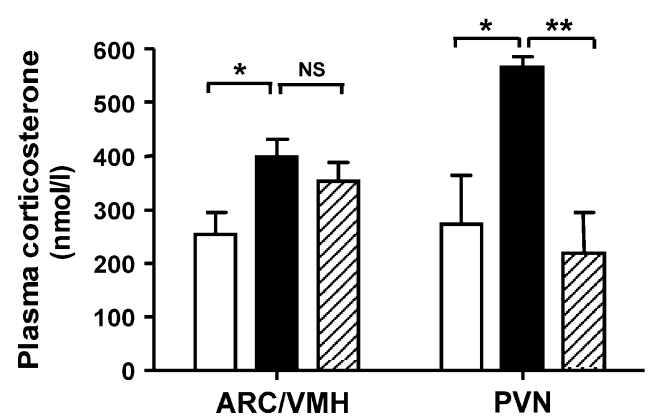

C

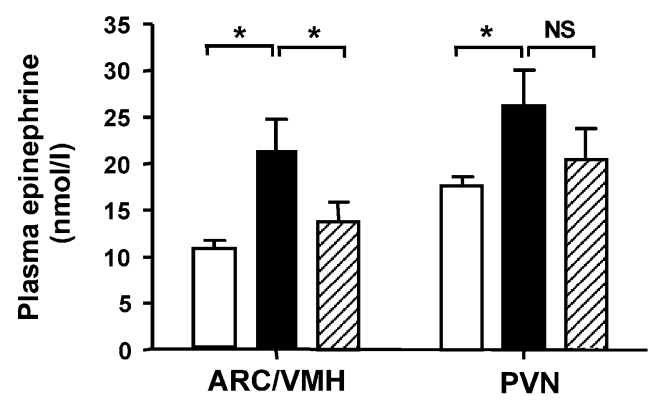

b
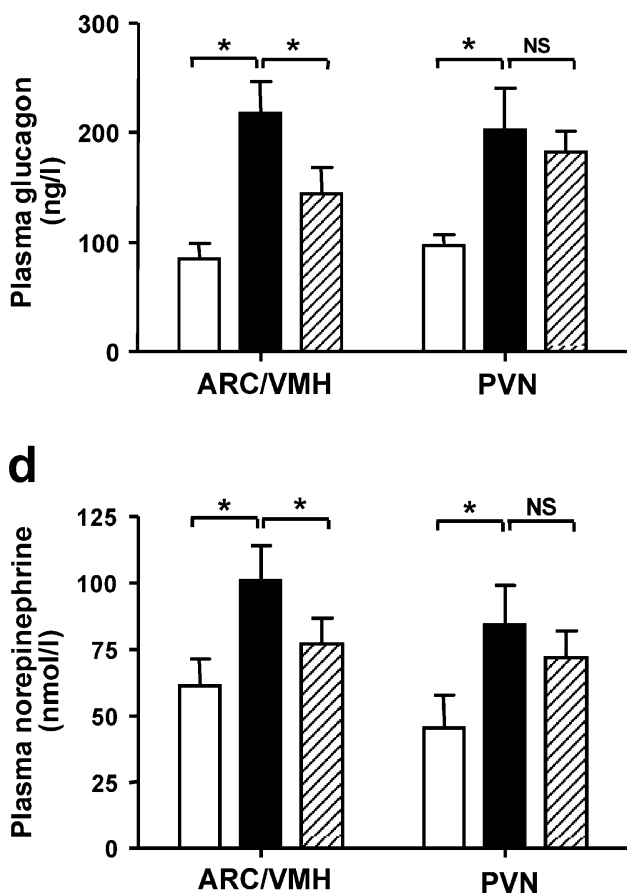
poglycaemia. Overexpression of DN-AMPK in the ARC/ VMH and PVN did not significantly alter AMPK activity in the other hypothalamic nuclei (data not shown).

Hypothalamic AMPK inhibition attenuates counter-regulatory responses Systemic hypoglycaemia increased plasma concentrations of corticosterone, glucagon, epinephrine and norepinephrine (Table 2). Prior i.c.v. administration of the AMPK inhibitor compound C, however, significantly attenuated hypoglycaemia-induced changes in plasma corticosterone, glucagon and catecholamines (Table 2). Intracerebroventricular administration of compound $\mathrm{C}$ also reversed an increase in hypothalamic $\alpha 2$-AMPK activity induced by hypoglycaemia (Table 2 ).

Overexpression of DN-AMPK in both the ARC/VMH and PVN did not cause a significant change in basal counterregulatory hormone concentrations (data not shown). DNAMPK overexpression in the ARC/VMH significantly attenuated hypoglycaemia-induced changes in plasma glucagon and catecholamine levels, while it did not affect corticosterone response (Fig. 4a-d).

In contrast, DN-AMPK overexpression in the PVN prevented the hypoglycaemia-induced increase in plasma corticosterone levels. It tended to reduce catecholamine responses but had no effect on glucagon response (Fig. 4a-d).

\section{Discussion}

We have shown here that insulin-induced systemic hypoglycaemia caused hypothalamic AMPK activation. Extracellular brain glucose levels are approximately $0.5 \mathrm{mmol} / 1$ when plasma glucose levels fall to $2-3 \mathrm{mmol} / \mathrm{l}$ [21]. Decreased glucose levels in extracellular brain fluid may cause AMPK activation in most neurons by depleting cellular energy, as the brain has an absolute dependence on glucose as a fuel. On the other hand, i.p. administration of a large amount of insulin may also contribute to hypothalamic AMPK activation as i.c.v. infusion of insulin has been shown to increase AMPK phosphorylation [22].

Notably, hypoglycaemia-induced AMPK activation was confined to the hypothalamic ARC/VMH and PVN, while AMPK activities in the LH and SOX remained unchanged. Moreover, hypoglycaemia increased hypothalamic $\alpha 2-$ AMPK but not $\alpha 1$-AMPK activities, a result consistent with observations that only hypothalamic $\alpha 2$-AMPK activity was altered by administration of alpha-lipoic acid or leptin or by induction of diabetes [11, 12, 23]. Thus systemic hypoglycaemia appears to activate hypothalamic AMPK in a region- and isoform-specific manner.

In our study, inhibition of hypothalamic AMPK activity significantly attenuated counter-regulatory responses, resulting in severe and prolonged hypoglycaemia. Some of the animals died without recovering from hypoglycaemia. Furthermore, hypothalamic AMPK activation by i.c.v. AICAR increased plasma counter-regulatory hormone levels. Taken together, hypothalamic AMPK activation in- duced by systemic hypoglycaemia is critical for triggering protective counter-regulatory responses and thereby recovering from hypoglycaemia.

In contrast to these findings, intra-VMH injection of AICAR has recently been reported to increase hepatic glucose production during a hypoglycaemic clamp, but without significant increases in plasma concentrations of glucagon and catecholamine [24]. In line with this, i.c.v. administration of AICAR during hypoglycaemia did not further increase plasma counter-regulatory hormone levels in our study. Hypothalamic AMPK activity in a hypoglycaemic condition would be already at a high level. Thus i.c.v. AICAR might be unable to increase AMPK activity sufficiently for stimulation of counter-regulatory responses.

The VMH is thought to play a critical role in sensing hypoglycaemia and initiating counter-regulatory hormonal responses. VMH neuroglycopenia causes the release of the early counter-regulatory responders, glucagon and catecholamines [3]. Conversely, the introduction of VMH lesions or direct delivery of glucose into the VMH suppresses these responses to systemic hypoglycaemia $[4,25]$. In the present study, DN-AMPK overexpression in the $\mathrm{ARC} / \mathrm{VMH}$ did not significantly alter basal counterregulatory hormone levels but did attenuate the hypoglycaemia-induced increase in plasma glucagons and catecholamine concentrations. The rats overexpressing DN-AMPK in their ARC/VMH had more severe hypoglycaemia during its early phase but no delay in recovery from hypoglycaemia. These results suggest that AMPK activation in the ARC/VMH may mediate glucagon and catecholamine responses, which are important for an early counter-regulation.

In the present study, the PVN was another site showing AMPK activation in response to hypoglycaemia. DNAMPK overexpression in the PVN inhibited corticosterone response to hypoglycaemia. It also tended to decrease hypoglycaemia-induced catecholamine responses but had no effect on glucagon response. In these rats, a recovery from hypoglycaemia was significantly delayed, whereas there was no difference in plasma glucose concentrations in the early phase. Some animals failed to recover from hypoglycaemia and died. These results suggest that AMPK activation in the PVN may be important for activation of the hypothalamo-pituitary-adrenal axis and late counterregulatory responses.

Consistent with these findings, it has been shown that PVN neurons are activated by systemic hypoglycaemia [26]. PVN activation causes activation of the hypothalamo-pituitary-adrenal axis by releasing corticotrophinreleasing factor and vasopressin [27]. It may also activate the sympathetic nervous system, as PVN neurons project to autonomic pre-ganglionic cells in the spinal cord [28]. Repeated hypoglycaemia has been shown to reduce counter-regulatory responses, a phenomenon known as hypoglycaemia-associated autonomic failure (HAAF) [29]. PVN activation is suppressed by repeated hypoglycaemia and inactivation of PVN neurons during hypoglycaemia 
can cause HAAF [30]. Therefore, it will be interesting to study if repeated hypoglycaemia can cause a reduction in PVN AMPK activation and if a manoeuvre activating PVN AMPK can prevent HAAF.

Several questions remain to be answered. Glucosesensitive neurons are present in the brainstem, and local neuroglycopenia in the ponto-medullary junction has been found to induce hyperphagic responses and elevate bloodglucose levels [31]. Thus it will be interesting to determine if AMPK activation in brainstem regions is involved in this process. In addition, recent studies have indicated that the hypothalamic K-ATP channel plays an important role in mediating counter-regulatory responses $[32,33]$. A possible relationship between the K-ATP channel and AMPK has been proposed [6] and needs to be clarified. Finally, the downstream mechanism by which hypothalamic AMPK activation induces counter-regulatory responses is unknown, and additional studies are necessary to identify it.

Acknowledgements This work was supported by a National Research Laboratory grant from the Korean Ministry of Health \& Welfare (03-PJ1-PG1-CH05-0005), the Korean Ministry of Science and Technology (M1040000000804J000000810), and the Asan Institute of Life Sciences (04-326). The authors thank J. Ha (Kyunghee University) for providing the DN-AMPK-expressing plasmids.

\section{References}

1. Cryer PE (1981) Glucose counterregulation in man. Diabetes 30:261-264

2. Biggers DW, Myers SR, Neal D et al (1989) Role of brain in counterregulation of insulin-induced hypoglycemia in dogs. Diabetes 3:7-16

3. Borg WP, Sherwin RS, During MJ, Borg MA, Shulman GI (1995) Local ventromedial hypothalamus glucopenia triggers counterregulatory hormone release. Diabetes 44:180-184

4. Borg MA, Sherwin RS, Borg WP, Tamborlane WV, Shulman GI (1997) Local ventromedial hypothalamus glucose perfusion blocks counterregulation during systemic hypoglycemia in awake rats. J Clin Invest 99:361-365

5. Hardie DG, Carling D, Carlson M (1998) The AMP-activated/ SNF1 protein kinase subfamily: metabolic sensors of the eukaryotic cell? Annu Rev Biochem 67:821-855

6. Rutter GA, da Silva Xavier G, Leclerc I (2003) Roles of 5'AMP-activated protein kinase (AMPK) in mammalian glucose homeostasis. Biochem J 375:1-16

7. Mu J, Brozinick JT Jr, Valladares O, Bucan M, Birnbaum MJ (2001) A role for AMP-activated protein kinase in contractionand hypoxia-regulated glucose transport in skeletal muscle. Mol Cell 7:1085-1094

8. Henin N, Vincent MF, Gruber HE, Van den Berghe G (1995) Inhibition of fatty acid and cholesterol synthesis by stimulation of AMP-activated protein kinase. FASEB J 9:541-546

9. da Silva Xavier G, Leclerc I, Salt IP et al (2000) Role of AMPactivated protein kinase in the regulation by glucose of islet beta cell gene expression. Proc Natl Acad Sci U S A 97:40234028

10. Turnley AM, Stapleton D, Mann RJ, Witters LA, Kemp BE, Bartlett PF (1999) Cellular distribution and developmental expression of AMP-activated protein kinase isoforms in mouse central nervous system. J Neurochem 72:1707-1716
11. Kim MS, Park JY, Namkoong C et al (2004) Anti-obesity effects of alpha-lipoic acid mediated by suppression of hypothalamic AMP-activated protein kinase. Nat Med 10:727733

12. Minokoshi Y, Alquier T, Furukawa N et al (2004) AMP-kinase regulates food intake by responding to hormonal and nutrient signals in the hypothalamus. Nature 428:569-574

13. Kim MS, Small CJ, Stanley SA et al (2000) The central melanocortin system affects the hypothalamo-pituitary thyroid axis and may mediate the effect of leptin. J Clin Invest 105:1005-1011

14. Stein SC, Woods A, Jones NA, Davison MD, Carling D (2000) The regulation of AMP-activated protein kinase by phosphorylation. Biochem J 345:437-443

15. Mu J, Barton ER, Birnbaum MJ (2003) Selective suppression of AMP-activated protein kinase in skeletal muscle: update on 'lazy mice'. Biochem Soc Trans 31:236-241

16. Mercer EH, Hoyle GW, Kapur RP, Brinster RL, Palmiter RD (1991) The dopamine beta-hydroxylase gene promoter directs expression of E. coli lac $Z$ to sympathetic and other neurons in adult transgenic mice. Neuron 7:703-716

17. Obici S, Feng Z, Arduini A, Conti R, Rossetti L (2003) Inhibition of hypothalamic carnitine palmitoyltransferase-1 decreases food intake and glucose production. Nat Med 9: 756-761

18. Paxinos G, Watson C (1997) The rat brain in stereotaxic coordinates, 2nd edn. Academic, San Diego, CA, p. 25-31 plates

19. Davies SP, Carling D, Hardie DG (1989) Tissue distribution of the AMP-activated protein kinase, and lack of activation by cyclic-AMP-dependent protein kinase, studied using a specific and sensitive peptide assay. Eur J Biochem 186:123-128

20. Kim MS, Yoon CY, Jang PG et al (2004) The mitogenic and anti-apoptotic actions of ghrelin in 3T3-L1 adipocytes. Mol Endocrinol 18:2291-2301

21. Silver IA, Erecinska M (1994) Extracellular glucose concentration in mammalian brain: continuous monitoring of changes during increased neuronal activity and upon limitation in oxygen supply in normo-, hypo-, and hyperglycemic animals. J Neurosci 14:5068-5076

22. Perrin C, Knauf C, Burcelin R (2004) Intracerebroventricular infusion of glucose, insulin, and the adenosine monophosphateactivated kinase activator, 5-aminoimidazole-4-carboxamide-1beta-D-ribofuranoside, controls muscle glycogen synthesis. Endocrinology 145:4025-4033

23. Namkoong C, Kim MS, Jang PG et al (2005) Enhanced hypothalamic AMP-activated protein kinase activity contributes to hyperphagia in diabetic rats. Diabetes 54:63-68

24. McCrimmon RJ, Fan X, Ding Y, Zhu W, Jacob RJ, Sherwin RS (2004) Potential role for AMP-activated protein kinase in hypoglycemia sensing in the ventromedial hypothalamus. Diabetes 53:1953-1958

25. Borg WP, During MJ, Sherwin RS, Borg MA, Brines ML, Shulman GI (1994) Ventromedial hypothalamic lesions in rats suppress counterregulatory responses to hypoglycemia. J Clin Invest 93:1677-1682

26. Niimi M, Sato M, Tamaki M, Wada Y, Takahara J, Kawanishi K (1995) Induction of Fos protein in the rat hypothalamus elicited by insulin-induced hypoglycemia. Neurosci Res 23:361-364

27. Herman JP, Cullinan WE (1997) Neurocircuitry of stress: central control of the hypothalamo-pituitary-adrenocortical axis. Trends Neurosci 20:78-84

28. Hosoya Y, Sugiura Y, Okado N, Loewy AD, Kohno K (1991) Descending input from the hypothalamic paraventricular nucleus to sympathetic preganglionic neurons in the rat. Exp Brain Res 85:10-20

29. Cryer PE (2001) Hypoglycemia-associated autonomic failure in diabetes. Am J Physiol Endocrinol Metab 281:E1115-E1121 
30. Evans SB, Wilkinson CW, Bentson K, Gronbeck P, Zavosh A, Figlewicz DP (2001) PVN activation is suppressed by repeated hypoglycemia but not antecedent corticosterone in the rat. Am J Physiol Regul Integr Comp Physiol 281:R1426-R1436

31. Ritter S, Dinh TT, Zhang Y (2000) Localization of hindbrain glucoreceptive sites controlling food intake and blood glucose. Brain Res 856:37-47
32. Evans ML, McCrimmon RJ, Flanagan DE et al (2004) Hypothalamic ATP-sensitive $\mathrm{K}^{+}$channels play a key role in sensing hypoglycemia and triggering counterregulatory epinephrine and glucagon responses. Diabetes 53:2542-2551

33. Miki T, Liss B, Minami K et al (2001) ATP-sensitive $\mathrm{K}^{+}$ channels in the hypothalamus are essential for the maintenance of glucose homeostasis. Nat Neurosci 4:507-512 